\title{
Moderately differentiated esophageal squamous cell carcinoma has a poor prognosis after neoadjuvant chemoradiotherapy
}

\author{
Wenwu He ${ }^{1 \#}$, Tianqin Mao ${ }^{2 \#}$, Jiaxin Yan ${ }^{3 \#}$, Xuefeng Leng ${ }^{1}$, Xuyang Deng ${ }^{1}$, Qin Xie ${ }^{1}$, Lin Peng ${ }^{1}$, \\ Qiong Liao ${ }^{3}$, Marco Scarpa $^{4}$, Yongtao Han ${ }^{1}$
}

${ }^{1}$ Department of Thoracic Surgery, Sichuan Cancer Hospital \& Research Institute, School of Medicine, University of Electronic Science and Technology of China (UESTC), Chengdu, China; ${ }^{2}$ School of Medicine, University of Electronic Science and Technology of China (UESTC), Chengdu, China; ${ }^{3}$ Department of Pathology, Sichuan Cancer Hospital \& Research Institute, School of Medicine, University of Electronic Science and Technology of China (UESTC), Chengdu, China; ${ }^{4}$ Clinica Chirurgica I Unit, Azienda Ospedaliera di Padova, Padova, Italy

Contributions: (I) Conception and design: W He, T Mao, J Yan; (II) Administrative support: Y Han, L Peng; (III) Provision of study materials or patients: W He, X Leng, Q Liao; (IV) Collection and assembly of data: X Deng, Q Xie; (V) Data analysis and interpretation: T Mao, M Scarpa; (VI) Manuscript writing: All authors; (VII) Final approval of manuscript: All authors.

\#These authors contributed equally to this work.

Correspondence to: Yongtao Han, MD, FRCS. Department of Thoracic Surgery, Sichuan Cancer Hospital \& Research Institute, School of Medicine, University of Electronic Science and Technology of China (UESTC), Chengdu, China. Email: yongtao_han@126.com.

Background: Neoadjuvant chemoradiotherapy (NCRT) plus surgery is the standard treatment for esophageal squamous cell carcinoma (ESCC); however, further analysis is needed to detail the histopathological characteristics of ESCC and their clinical significance after NCRT. This study aimed to present the pathological characteristics of ESCC and their association with prognosis after NCRT.

Methods: All patients with ESCC who underwent NCRT followed by surgical resection at Sichuan Cancer Hospital (China) from January 2018 to December 2019 were included. Resection specimens of both the primary disease and lymph nodes were re-evaluated by an experienced pathologist. After NCRT, the pathological characteristics of the residual tumor were evaluated based on the Japanese residual tumor pattern, Mandard tumor regression grade (Mandard-TRG), local inflammatory infiltration classification, and lymph node status.

Results: Among the 103 patients with ESCC included in this study, the pathological complete response (pCR) rate was 34\% (35/103). The pCR rate of patients with poorly differentiated tumors (31/72) was higher $(43.1 \%)$ than that of patients with well or moderately differentiated tumors $(\mathrm{P}<0.05)$. The residual tumor rate was $66 \%(68 / 103)$. A positive correlation was noted between the Japanese residual tumor pattern and Mandard-TRG (Kendall's tau-b =0.857, $\mathrm{P}<0.001$ ). Tumor infiltration depth, lymph node positivity, moderate differentiation, and tumor recurrence were associated with poor oncological outcomes $(\mathrm{P}<0.05)$.

Conclusions: Patients with poorly differentiated tumors can obtain an excellent short-term response; however, they have extremely poor long-term survival. For patients with moderately differentiated tumors, both the short- and long-term outcomes are poor. Lymph node status after NCRT is a prognostic factor for ESCC treated with NCRT.

Keywords: Pathological characteristics; neoadjuvant chemoradiotherapy (NCRT); oncological outcome; esophageal squamous cell carcinoma (ESCC)

Submitted Jan 26, 2021. Accepted for publication Apr 22, 2021.

doi: $10.21037 / \mathrm{atm}-21-1815$

View this article at: http://dx.doi.org/10.21037/atm-21-1815 


\section{Introduction}

Neoadjuvant chemoradiotherapy (NCRT) plus surgery has become the standard treatment for patients with esophageal squamous cell carcinoma (ESCC) in the last decade, because it can increase the $\mathrm{R} 0$ resection rate and improve the overall survival (OS) $(1,2)$. As a result, changes in pathological features of the primary disease and lymph nodes in response to NCRT, as well as their prognostic value, have attracted the attention of some researchers. The rate of pathological complete response ( $\mathrm{pCR}$; no vital tumor cells in resected specimens or lymph nodes) after NCRT ranges from $27 \%$ to $49 \%(1-5)$, indicating that more than half of patients have persistent residual disease after NCRT. Recent evidence suggests that patients with ESCC who reach pCR after NCRT have longer median OS than those with residual tumor (6). Therefore, it is essential that the correlation between pathological changes and prognosis in patients with pathological residual tumor (pRT) is investigated to inform effective clinical management.

Research on residual tumor patterns in ESCC after neoadjuvant chemotherapy revealed that shallow remnants are dominant, and that deep and diffuse remnants are associated with metastasis (7). Also, the primary tumor regression grade (TRG) and lymph node status are considered valuable prognostic factors in patients with esophageal cancer who receive neoadjuvant chemotherapy $(8,9)$. Patients with a good response on nodal metastasis have better survival outcomes irrespective of the TRG of the primary disease (10). However, a lymph node with stable disease is associated with lower disease-free survival than N0 in ESCC after NCRT (11).

The pathological characteristics of the primary tumor or lymph nodes have been shown to have prognostic value following NCRT. However, although the pathological characteristics of locally advanced ESCC after neoadjuvant chemotherapy have been reported (12), comprehensively pathological changes after NCRT and their prognostic value in ESCC remain unclear. Therefore, in this study, we re-evaluated the pathological parameters of patients with ESCC following NCRT on the basis of the Japanese pattern of residual tumor, Mandard-TRG, local inflammatory cell infiltration classification, and lymph node status in order to present the pathological characteristics of ESCC after NCRT and their association with patient survival.

We present the following article in accordance with the STROBE reporting checklist (available at http://dx.doi. org/10.21037/atm-21-1815).

\section{Methods}

\section{Patients}

All the consecutive patients who were diagnosed with locally advanced ESCC and underwent NCRT followed by radical esophagectomy at the Sichuan Cancer Hospital, China, between January 2018 and December 2019 were included. The inclusion criterion was histologically confirmed ESCC with pathological stage ypT0-4aN02M0. All patients underwent NCRT followed by surgery and had a European Cooperative Oncology Group performance status score of $0-2$. Patients with a history of local or distant metastasis were excluded, as were those who underwent anticancer treatments other than NCRT. All patients were administered platinum combined with paclitaxel, docetaxel, capecitabine, or fluorouracil and concurrent radiotherapy. A total dose of 40 Gy was administered in 20 fractions of 2 Gy. Radical esophagectomy was performed 5 to 6 weeks after the completion of NCRT. All procedures performed in this study involving human participants were in accordance with the Declaration of Helsinki (as revised in 2013). This study was approved by the Ethical Committee of the Sichuan Cancer Hospital (No. SCCHEC-02-2017-043), and informed consent was obtained from all participants.

\section{Patbological reassessment}

Initially, all resected specimens were processed using the standard protocol for pathological examination (13), which included the assessment of tumor location, size, differentiation, and stage. To characterize the pathological parameters of the primary tumor and lymph nodes after NCRT, all specimens were re-evaluated by an experienced pathologist. We defined pCR as the absence of vital tumor cells either in the primary disease or in the resected lymph nodes. Thereafter, the Japanese pattern of residual tumor (7) was used to gain insight into the position of the residual cancer in the esophageal wall after NCRT. According to this classification, there are 4 patterns of residual tumor: type 1 (shallow remnant): the residual tumor exists only in the mucosa or in the mucosal and submucosal layers; type 2 (central remnant): the residual tumor is present in the submucosa or in the submucosa and muscularis propria; type 3 (deep remnant): the residual tumor is present in the muscularis propria or in the muscularis and adventitia; and type 4 (diffuse remnant): the residual tumor cells are diffuse in the esophageal wall (for instance, residual tumor cells 

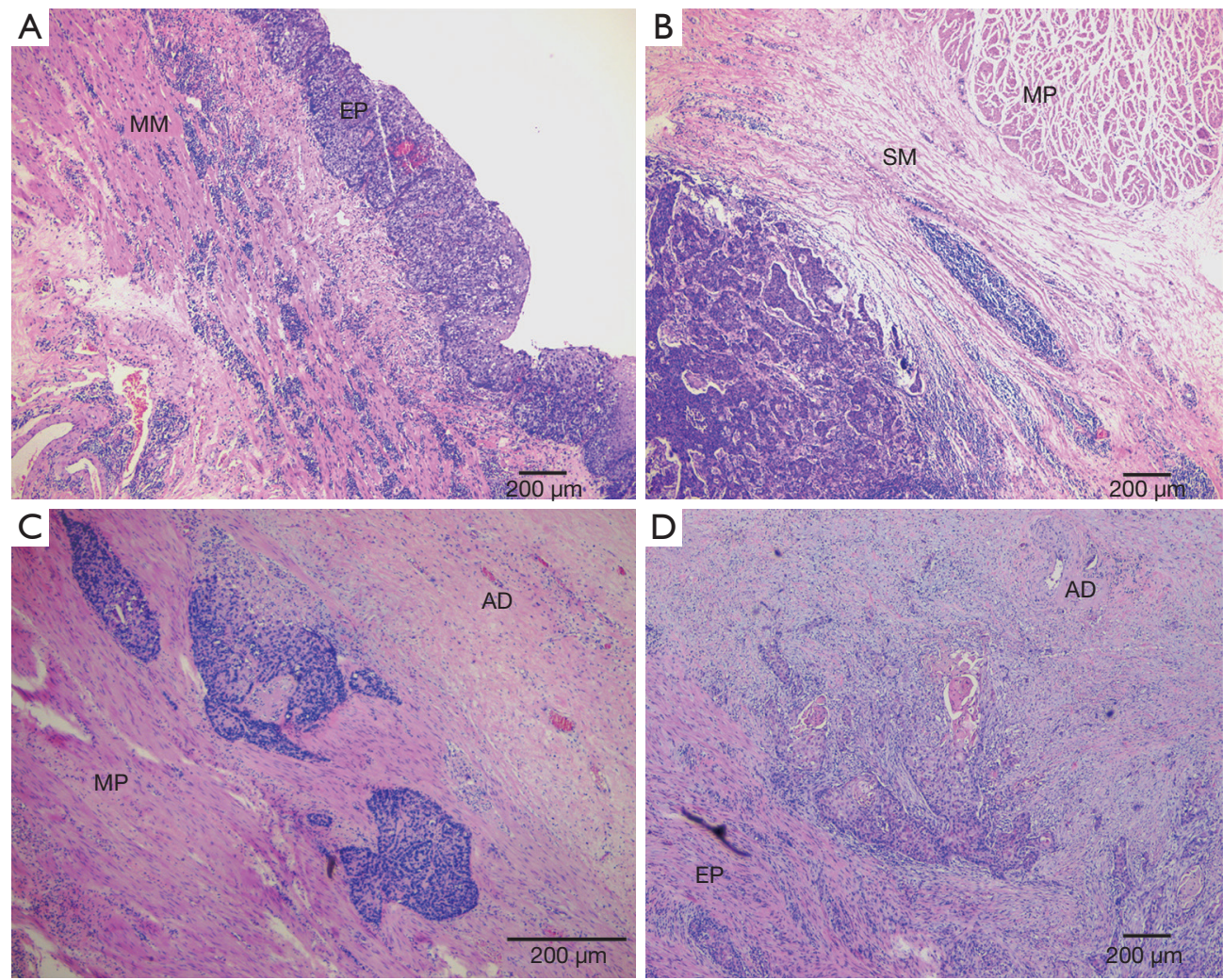

Figure 1 Four categories of Japanese residual tumor pattern in esophageal wall tissue after NCRT using HE staining. (A) Type 1 (shallow remnant within EP to MM layers); (B) type 2 (central remnant within SM to MP layers); (C) type 3 (deep remnant within MP to AD layers); (D) type 4 (diffuse remnant from EP to AD layers). Scale bars in images, $200 \mu \mathrm{m}$. NCRT, neoadjuvant chemoradiotherapy; EP, epithelium; $\mathrm{MM}$, muscularis mucosa; $\mathrm{SM}$, submucosa; MP, muscularis propria; $\mathrm{AD}$, adventitia.

are found in the mucosa to the muscularis propria, from the mucosa to the adventitia, or from the submucosa to the adventitia) (7) (Figure 1). To facilitate further analysis, an absence of residual tumor cells at the site of the primary tumor was characterized as type 0 in our study.

The TRG after NCRT was re-evaluated using the Mandard-TRG system as follows (14): TRG 1: an absence of tumor cells (complete regression); TRG 2: isolated residual tumor cells scattered through the fibrosis; TRG 3: an increased number of residual tumor cells but with fibrosis still predominant; TRG 4: the presence of residual tumor cells which outgrow the fibrosis; and TRG 5, an absence of evidence of regression (Figure 2).

Based on the degree of inflammatory cell infiltration per microscopic field, inflammation in the primary tumor was classified into four grades: no infiltration: an absence of inflammatory cells throughout the whole specimen; low infiltration: inflammatory cells accounting for less than one-third of the cells in each field; moderate filtration: inflammatory cells accounting for between one-third and two-thirds of the cells in each field; and high infiltration: inflammatory cells accounting for more than two-thirds of the cells in each field.

\section{Follow-up}

The main methods of follow-up were telephone calls, outpatient visits, and hospitalization review. After surgery, patients were followed-up every 3 months during the first year and every 6 months during the second year. Diagnostic examination was performed only in cases of suspected recurrence. Locoregional recurrence was defined as recurrence in the tumor bed or anastomotic recurrence at the primary disease site or regional lymph nodes. Distant 

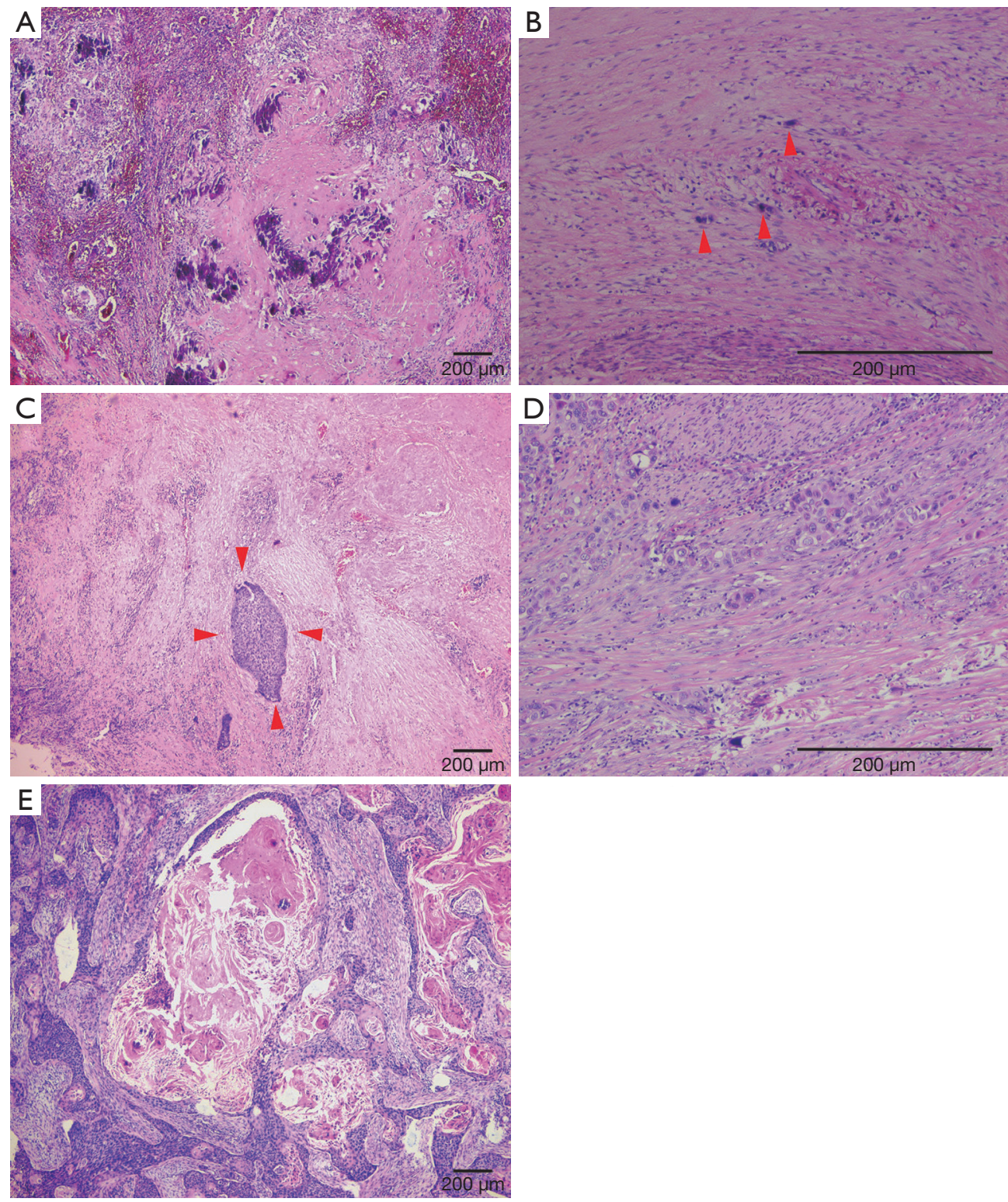

Figure 2 Histological images of each Mandard-TRG by HE staining. (A) TRG 1 (no residual tumor cells, tumor complete regression with significant calcification); (B) TRG 2 (single residual tumor cell scattered through the fibrosis); (C) TRG 3 (an increased number of residual tumor cells but cells outgrown by fibrosis); (D) TRG 4 (residual tumor cells outgrowing fibrosis); (E) TRG 5 (no evidence of tumor regression). Scale bars in images, $200 \mu \mathrm{m}$. TRG, tumor regression grade.

metastasis was defined as metastasis to other organs.

\section{Statistical analysis}

All statistical analyses were performed using IBM SPSS Statistics for Windows, version 25.0 (IBM, Corp., Armonk, NY, USA). Categorical variables were presented as frequencies and compared using Pearson's chi-square test and Fisher's exact test according to the cell counts. Ordered categorical variables were compared using the KruskalWallis test. Correlations between groups were assessed using Kendall's tau-b. OS was defined as the time from the date of surgery to the date of death or the last patient contact. Progression-free survival (PFS) was defined as the 
time from the date of surgery to recurrence, any relapse, or death. Survival was analyzed using Kaplan-Meier curves. A two-sided $\mathrm{P}$ value of $<0.05$ was considered to be statistically significant.

\section{Results}

\section{Patient characteristics}

A total of 103 patients who underwent NCRT followed by surgery were enrolled. Among the 103 patients, 93 (90.3\%) were male and $59(57.3 \%)$ were $\geq 60$ years old. In $13(12.6 \%)$, $57(55.3 \%)$, and $33(32 \%)$ cases, the tumor was located in the upper, middle, and lower third of the esophagus, respectively. Based on histopathological data, the majority of patients $(72,69.9 \%)$ had poorly differentiated tumors. A total of 35 (34\%) patients achieved pCR, and 68 (66\%) patients had pRT (Table 1).

\section{Differentiation and inflammation classification in patients with $p C R$}

The pCR rate for patients with poorly differentiated tumors was $43.1 \%$ (31/72), which was higher than the pCR rates among patients with well and moderately differentiated tumors $(\mathrm{P}=0.002)$. Interestingly, the rate of $\mathrm{pCR}$ declined with the grade of inflammation, except in 1 case with no inflammatory response. However, no significant correlation was noted between the inflammation classification and pCR rate (Table 2).

\section{Pathological features of patients with $p R T$}

A total of 68 patients had pRT, including 4 (3.9\%) patients who achieved a pCR in the primary tumor but not in the lymph node (ypT0N+). According to the Japanese pattern of residual tumor, there were 18 (26.5\%), $16(23.5 \%)$, $6(8.8 \%)$, and $24(35.3 \%)$ patients with type 1, 2, 3, and 4 patterns, respectively, after NCRT (Table 1). With respect to TRG, 39 (37.9\%), 20 (19.4\%), 24 (23.3\%), 17 (16.5\%), and 3 (2.9\%) patients had TRG 1, 2, 3, 4, and 5, respectively. The relationship between the Japanese pattern and Mandard-TRG was assessed using Kendall's tau-b, and the results revealed a strong positive correlation between them (Kendall's tau-b $=0.857, \mathrm{P}<0.001$ ). Low and moderate inflammatory infiltration was dominant in all resected specimens, especially in those with TRG 1.

\section{Lymph nodes}

The median lymph node yield was 17 (range: 2-41). Persistent tumor metastasis was noted in 53 lymph nodes of $28(27.2 \%)$ patients. Among the patients with lymph node metastasis, the mean ratio of positive to total lymph nodes was $13.3 \%$ (range: $4-33 \%$ ). Also, we analyzed the correlations of other pathological parameters with lymph node metastasis. Lymph node metastasis was not found to be significantly correlated with sex, age, or tumor location, but it was significantly correlated with the degree of differentiation (Table 3). The lymph node metastasis rate was higher in moderately differentiated tumors than in well or poorly differentiated tumors $(\mathrm{P}<0.001)$. Furthermore, the primary TRG was discovered to be associated with lymph node metastasis (Kendall's tau-b $=0.315, \mathrm{P}=0.001$ ). The rates of lymph node metastasis with different MandardTRG grades were as follows: TRG 1 (4 patients, 10.3\%), TRG 2/3 (14 patients, 31.8\%); and TRG 4/5 (10 patients, $50 \%)$. This observation indicated that the higher the TRG, the more likely lymph node metastasis was to occur $(\mathrm{P}=0.003)$. No significant difference was noted in the recurrence rate between the positive lymph node and negative lymph node groups ( $39.3 \%$ vs. $21.3 \%, \mathrm{P}=0.08$ ).

\section{Oncological outcomes}

The median follow-up duration for all patients was 17 (range: 2-29) months. We analyzed OS and PFS in patients according to different pathological characteristics. The 1-year OS and PFS rates of all patients were $76.7 \%$ and $67 \%$, respectively. No significant differences were noted between patients with pCR and those with pRT in terms of OS $(82.9 \%$ vs. $73.5 \%, \log$-rank $\mathrm{P}=0.303)$ or PFS (71.4\% vs. $64.7 \%$, log-rank $\mathrm{P}=0.428$ ) (Figure $3 A, B)$. Although no significant difference was noted in OS (types $1 / 2 / 3 / 4=88.9 \% / 75 \% / 66.7 \% / 62.5 \%, \log$-rank $\mathrm{P}=0.216$ ) among patients with different residual tumor patterns, a significant difference in PFS was observed (types 1/2/3/4 $=88.9 \% / 62.5 \% / 50 \% / 50 \%$, log-rank $\mathrm{P}=0.048$ ) (Figure 3C). Moreover, a significant difference was noted in the 1-year PFS rates of patients with different tumor differentiation grades (well/moderate/poor $=100 \% / 38.1 \% / 70.8 \%, \log$-rank $\mathrm{P}=0.001$ ) (Figure 3D). Further, we analyzed the OS and PFS curves of patients grouped according to lymph node status. The outcomes in the negative lymph node group were significantly better than those in the positive lymph node 
Page 6 of 12

He et al. Pathological features after NCRT in ESCC

Table 1 Pathological characteristics of patients and their correlation with survival status ( $\mathrm{n}=103)$

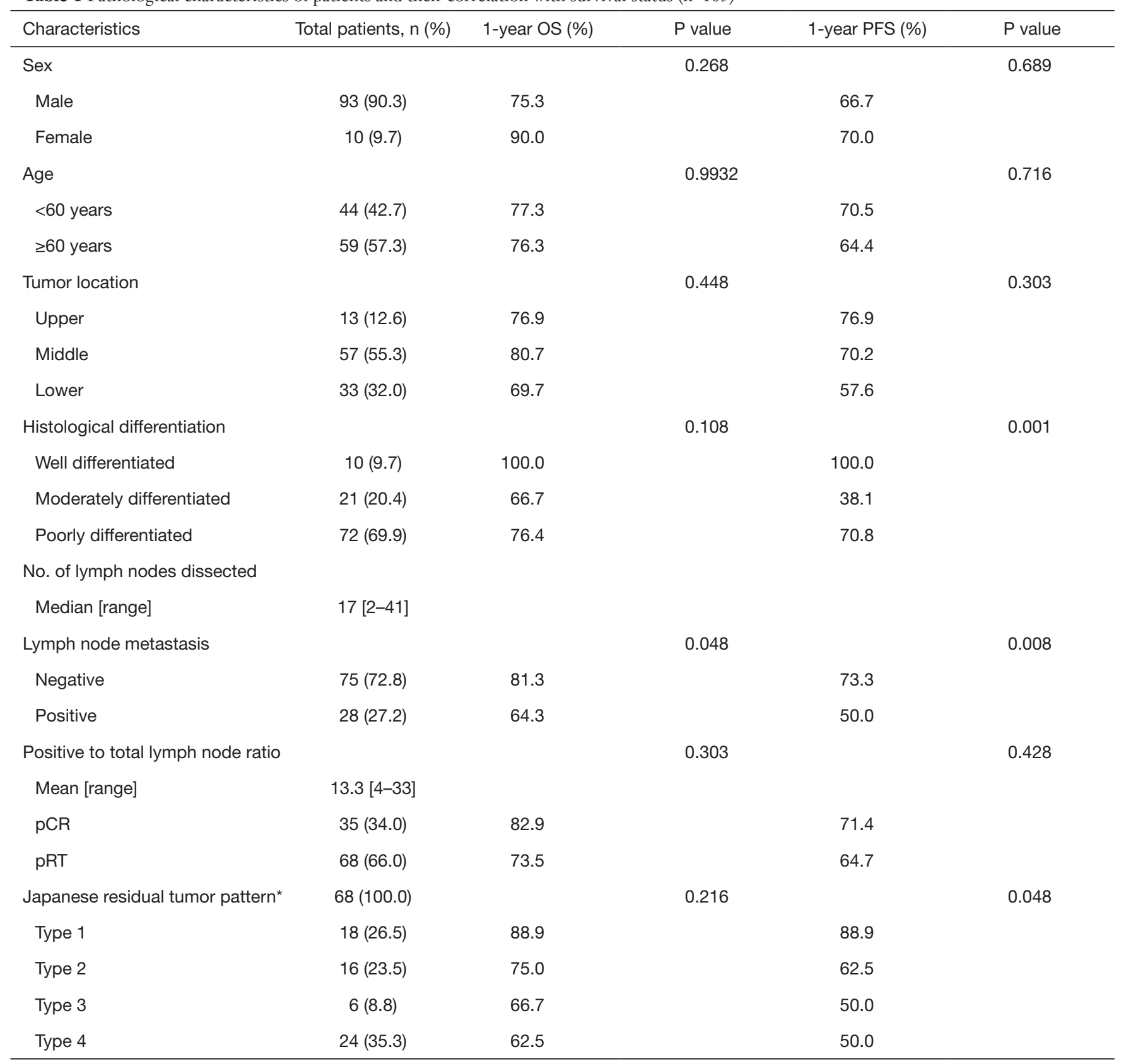

${ }^{*}$, four patients who achieved a complete response in the primary tumor but had residual disease in lymph nodes (ypTON+) were excluded. OS, overall survival; PFS, progression-free survival; pCR, pathological complete response; $p R T$, pathological residual tumor.

group, with the 1-year OS rates in the negative and positive lymph node groups being $81.3 \%$ and $64.3 \%$, respectively $(\mathrm{P}=0.048)$, and the 1 -year PFS rates being $73.3 \%$ and $50 \%$, respectively $(\mathrm{P}=0.008)$ (Figure $3 E, F)$.

Locoregional recurrence or distant metastasis was recorded in 27 of 103 (26.2\%) patients. Patients who experienced recurrence or metastasis had poorer 1-year OS than patients without recurrence or metastasis (metastasis/no metastasis $=37 \% / 90.8 \%$, log-rank $\mathrm{P}<0.001)$. The median OS for patients with recurrence or metastasis was only 12 months. There were no cases of recurrence or metastasis in a welldifferentiated tumor in this cohort. Recurrence or metastasis occurred in 11 of $21(52.4 \%)$ patients with moderately differentiated tumors and in 16 of $72(22.2 \%)$ patients with 
Table 2 Tumor response was related to the pathological characteristics of the primary tumor

\begin{tabular}{lccc}
\hline Characteristics & $\mathrm{pCR}(\mathrm{n}=35), \mathrm{n}(\%)$ & $\mathrm{pRT}(\mathrm{n}=68), \mathrm{n}(\%)$ & Total \\
\hline Histological differentiation & $3(30.0)$ & $7(70.0)$ & 10 \\
Well differentiated & $1(4.8)$ & $20(95.2)$ & 21 \\
Moderately differentiated & $31(43.1)$ & $41(56.9)$ & 72 \\
Poorly differentiated & & $24(60.0)$ & 40 \\
Inflammation classification* & $16(40.0)$ & $35(68.6)$ & 51 \\
Low infiltration & $16(31.4)$ & $8(72.7)$ & 11 \\
Moderate infiltration & $3(27.3)$ & & 0.660 \\
High infiltration & & & \\
\hline
\end{tabular}

*, one patient without any infiltration was excluded from the analysis due to the insufficient sample size. pCR, pathological complete response; pRT, pathological residual tumor.

Table 3 The relationships of pathological characteristics with lymph node status and recurrence/metastasis status

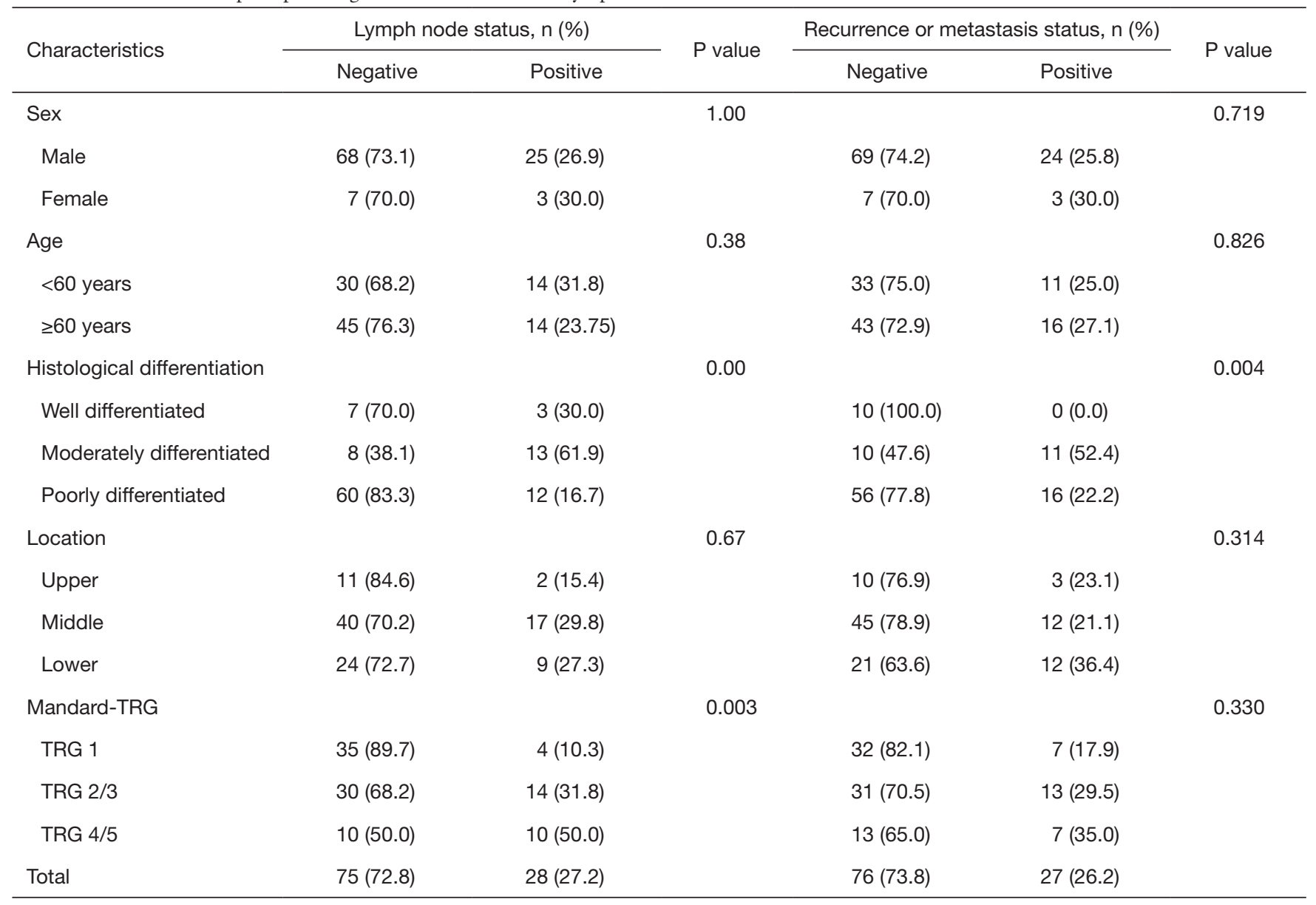

TRG, tumor regression grade. 

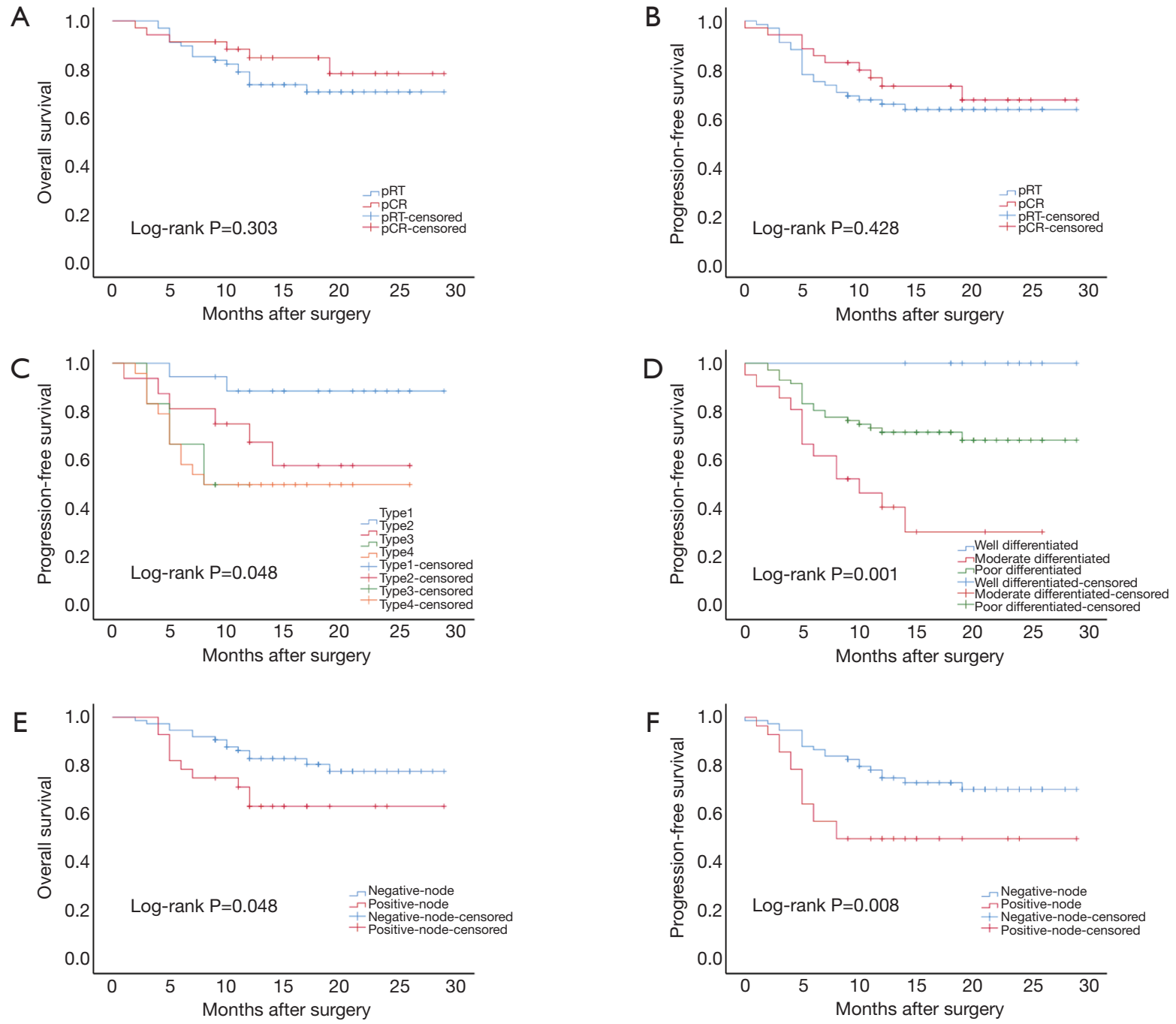

Figure 3 Kaplan-Meier OS and PFS. (A) OS in the pCR and pRT groups; (B) PFS in the pCR and pRT groups; (C) PFS of patients with different Japanese residual tumor patterns; (D) PFS of patients with different degrees of tumor differentiation; (E) OS of patients with and without lymph node metastasis; (F) PFS of patients with and without lymph node metastasis. Type 1: shallow remnant; type 2: central remnant; type 3: deep remnant; type 4: diffuse remnant. OS, overall survival; PFS, progression-free survival; pCR, pathological complete response; pRT, pathological residual tumor.

poorly differentiated tumors $(\mathrm{P}=0.004)$ (Table 3). Local recurrence $(6 / 11,54.5 \%)$ and distant metastasis $(5 / 11,45.5 \%)$ were each observed in approximately half of patients with moderately differentiated tumors. However, patients with poorly differentiated tumors were more likely to experience distant metastasis (11/16, 68.75\%).

\section{Discussion}

Study of the pathological characteristics and changes of
ESCC after NCRT is meaningful to providing information for the assessment of tumor response. In this study, we described in detail the pathological characteristics of ESCC after NCRT and their association with tumor treatment response and prognosis. We first assessed the pathological characteristics of patients with ESCC who received NCRT using the Japanese residual tumor pattern, Mandard-TRG system, local inflammatory infiltration classification, and lymph node status. We found that patients with poorly differentiated tumors showed a better response to NCRT 
than those with well or moderately differentiated tumors, which is in line with the findings of a previous study (15). A possible reason for this is that poorly differentiated tumors have a high turnover of cells, which increases their likelihood of being affected by DNA damage and apoptosis following NCRT (16). However, a previous study reported that poor tumor differentiation was an independent prognostic factor for recurrence after treatment in some solid tumors (17-19), suggesting that esophagectomy and surveillance are important after surgery in patients with pCR.

The present study reported that a high degree of inflammatory cell infiltration was associated with the carcinogenesis of esophageal cancer, which was similar with the results of previous studies $(20,21)$. Local inflammatory cell infiltration may provide important information regarding tumor response; however, its composition is complex. Local infiltration by inflammatory cells caused by proinflammatory cytokines induces, rather than tumor increases and tumor proliferation stimulating a high-grade local immune response, which may prevent further tumor dissemination and progression (22). In this study, low and moderate inflammatory cell infiltration was dominant in patients with $\mathrm{pCR}$, and 11 patients with high inflammatory infiltration and without TRG 4/5 achieved a good response in the primary tumor. Although no statistical significance was found, it can be conceivably hypothesized that before pCR, the degree of inflammatory cell infiltration in the primary tumor is high and gradually decreases after pCR. However, patients with a high level of infiltration may not achieve complete regression in the primary tumor; therefore, an interval of 5 to 6 weeks between the completion of NCRT and surgery is insufficient for obtaining a sufficient response to NCRT. A previous study reported that a time interval of 80 to 89 days between the completion of NCRT and surgery could significantly increase the odds of achieving pCR (23). Furthermore, recurrence/relapse was associated with high level of CD4+ $\mathrm{T}$ cell infiltration (24). In head and neck squamous cell carcinoma, the immunosuppressive milieu can be modified after chemoradiotherapy and is supposed to have a prognostic beneficial effect (25). Clarifying the relationship between inflammatory infiltration and tumor response to NCRT constitutes a considerable challenge, and it is necessary to perform large-sample studies to illustrate the correlation.

In the present study, the majority of patients $(66 \%)$ had residual disease after NCRT. Hence, one of the most significant current discussions about patients with pRT is the relationships between pathological parameters and prognosis. We found persistent nodal disease after NCRT in $4(3.9 \%)$ patients with pCR in the primary tumor (ypT0N+). The OS of these patients was lower than that of patients with pCR (26) and similar to that of patients with ypT+N+ (27). However, because this study enrolled a small number of patients, no significant difference was noted in 1 -year OS between patients with ypT0N+ and those with pCR or ypT+N+. Regarding the tumor residual pattern in the primary tumor, the dominant pattern in this study was diffuse remnant, followed by shallow remnant, central remnant, and deep remnant. Residual tumor cells were commonly present in adventitia, which is similar to the findings of recent studies on esophageal cancer treated with NCRT, which mostly comprised patients with esophageal adenocarcinoma (28). However, this finding is inconsistent with an observation made in a previous study, which reported that the mucosa and submucosa of the esophageal wall are commonly affected after chemoradiotherapy for esophageal cancer (29). The presence of residual tumor cells in the mucosa and submucosa increases the difficulty of diagnosing residual tumors after NCRT. Hence, the use of a TRG system to evaluate tumor response after NCRT is essential in clinical management. Many TRG systems exist (14,30-33); in this study, we selected the MandardTRG system to assess the resected specimens. The results revealed a strong positive correlation between the Japanese pattern of residual tumor and the Mandard-TRG system, indicating that deeper tumor invasion was associated with a worse response to NCRT in primary tumors.

In the present study, the rate of lymph node metastasis was $27.2 \%$, and the rate of lymph node metastasis was associated with differentiation and regression of the primary tumor. Lymph node metastasis is associated with poor prognosis (34), and lymph node down-staging after NCRT may improve prognosis $(10,35)$. In this analysis, patients with lymph node metastasis had shorter 1-year OS and PFS than those with negative lymph nodes; however, no significant differences were noted in the outcomes of patients with different TRGs, which indicated lymph node status to be a strong prognostic factor with greater importance than primary tumor regression. Moreover, it is critical for patients with positive lymph nodes to receive adjuvant treatment. A previous study reported that adjuvant treatment could significantly improve the median OS of patients with lymph node metastasis after surgery (36).

In the present study, we found that tumor differentiation was associated with lymph node metastasis, tumor 
recurrence, and distant metastasis. According to previous reports, poorly differentiated tumors should carry a high risk of both lymph node and distant metastases $(37,38)$; however, in the present study, moderately differentiated tumors were associated with high rates of lymph node metastasis and tumor recurrence, as well as poor PFS. A possible reason for this is that moderately differentiated tumors are insensitive to NCRT and have a high rate of lymph node metastasis and local recurrence or distant metastasis, which lead to moderately differentiated tumor with poor OS and PFS. Also, we found that the dominant type of metastasis in patients with poorly differentiated tumors was distant metastasis, which suggests that the diagnosis of micrometastases is a problem that urgently needs to be addressed. However, the accuracy of diagnosing micrometastases in ESCC can be improved with the application of technological developments such as liquid biopsy. Further, our results revealed that poorly differentiated tumors were associated with a better shortterm prognosis and a worse long-term prognosis than well or moderately differentiated tumor, while the prognosis for moderately differentiated tumors was poor in the short and long term. However, a large-sample clinical trial should be conducted to verify this inference.

Our results are subject to at least three limitations. First, this was a retrospective study conducted at a single institution. Hence, the representativeness of the results may be influenced by unmeasured and unknown confounders. Second, the number of patients enrolled was small; therefore, a large prospective study should be conducted to verify our findings. Finally, the follow-up time was too short to calculate a statistically significant correlation between survival and pathological features after NCRT.

\section{Conclusions}

Our study assessed the pathological characteristics of ESCC and their prognostic value after NCRT. The results showed that patients with poorly differentiated tumors can achieve an excellent short-term response but have dismal long-term survival, while those with moderately differentiated tumors have poor outcomes in both the short and the long term. Furthermore, lymph node status shows a higher prognostic value than primary tumor regression. For high-risk patients with ESCC, systematic assessment of the pathological characteristics after NCRT can provide important information regarding prognosis and facilitate treatment decision-making.

\section{Acknowledgments}

The authors thank Wei Xu for her assistance with the statistical analysis and interpretation. The authors also appreciate the academic support from AME Esophageal Cancer Collaborative Group.

Funding: This work was supported by Bethune Charitable Foundation (HX2020031).

\section{Footnote}

Reporting Checklist: The authors have completed the STROBE reporting checklist. Available at http://dx.doi. org/10.21037/atm-21-1815

Data Sharing Statement: Available at http://dx.doi. org/10.21037/atm-21-1815

Conflicts of Interest: All authors have completed the ICMJE uniform disclosure form (available at http://dx.doi. org/10.21037/atm-21-1815). The authors have no conflicts of interest to declare.

Ethical Statement: The authors are accountable for all aspects of the work in ensuring that questions related to the accuracy or integrity of any part of the work are appropriately investigated and resolved. All procedures performed in this study involving human participants were in accordance with the Declaration of Helsinki (as revised in 2013). This study was approved by the Ethical Committee of the Sichuan Cancer Hospital (No. SCCHEC-02-2017-043), and informed consent was obtained from all participants.

Open Access Statement: This is an Open Access article distributed in accordance with the Creative Commons Attribution-NonCommercial-NoDerivs 4.0 International License (CC BY-NC-ND 4.0), which permits the noncommercial replication and distribution of the article with the strict proviso that no changes or edits are made and the original work is properly cited (including links to both the formal publication through the relevant DOI and the license). See: https://creativecommons.org/ licenses/by-nc-nd/4.0/.

\section{References}

1. van Hagen P, Hulshof MC, van Lanschot JJ, et al. 
Preoperative chemoradiotherapy for esophageal or junctional cancer. N Engl J Med 2012;366:2074-84.

2. Yang H, Liu H, Chen Y, et al. Neoadjuvant chemoradiotherapy followed by surgery versus surgery alone for locally advanced squamous cell carcinoma of the esophagus (NEOCRTEC5010): a phase III multicenter, randomized, open-label clinical trial. J Clin Oncol 2018;36:2796-803.

3. Toxopeus EL, Nieboer D, Shapiro J, et al. Nomogram for predicting pathologically complete response after neoadjuvant chemoradiotherapy for oesophageal cancer. Radiother Oncol 2015;115:392-8.

4. Thomas M, Borggreve AS, van Rossum PSN, et al. Radiation dose and pathological response in oesophageal cancer patients treated with neoadjuvant chemoradiotherapy followed by surgery: a multiinstitutional analysis. Acta Oncol 2019;58:1358-65.

5. Hamai Y, Hihara J, Emi M, et al. Preoperative prediction of a pathologic complete response of esophageal squamous cell carcinoma to neoadjuvant chemoradiotherapy. Surgery 2018. [Epub ahead of print]. doi: 10.1016/ j.surg.2018.01.011.

6. Francoual J, Lebreton G, Bazille C, et al. Is pathological complete response after a trimodality therapy, a predictive factor of long-term survival in locally-advanced esophageal cancer? Results of a retrospective monocentric study. J Visc Surg 2018;155:365-74.

7. Hashimoto T, Makino T, Yamasaki M, et al. The pattern of residual tumor after neoadjuvant chemotherapy for locally advanced esophageal cancer and its clinical significance. Ann Surg 2020;271:875-84.

8. Davarzani N, Hutchins GGA, West NP, et al. Prognostic value of pathological lymph node status and primary tumour regression grading following neoadjuvant chemotherapy - results from the MRC OE02 oesophageal cancer trial. Histopathology 2018;72:1180-8.

9. Hatogai K, Fujii S, Kojima T, et al. Prognostic significance of tumor regression grade for patients with esophageal squamous cell carcinoma after neoadjuvant chemotherapy followed by surgery. J Surg Oncol 2016;113:390-6.

10. Davies AR, Myoteri D, Zylstra J, et al. Lymph node regression and survival following neoadjuvant chemotherapy in oesophageal adenocarcinoma. Br J Surg 2018;105:1639-49.

11. Leng $X, H e W$, Yang $H$, et al. Prognostic impact of postoperative lymph node metastases after neoadjuvant chemoradiotherapy for locally advanced squamous cell carcinoma of esophagus: from the results of
NEOCRTEC5010, a randomized multicenter study. Ann Surg 2019. [Epub ahead of print]. doi: 10.1097/ SLA.0000000000003727.

12. Nejman D, Livyatan I, Fuks G, et al. The human tumor microbiome is composed of tumor type-specific intracellular bacteria. Science 2020;368:973-80.

13. Klevebro F, Tsekrekos A, Low D, et al. Relevant issues in tumor regression grading of histopathological response to neoadjuvant treatment in adenocarcinomas of the esophagus and gastroesophageal junction. Dis Esophagus 2020;33:doaa005.

14. Mandard AM, Dalibard F, Mandard JC, et al. Pathologic assessment of tumor regression after preoperative chemoradiotherapy of esophageal carcinoma. Clinicopathologic correlations. Cancer 1994;73:2680-6.

15. Zhang X, Eyck BM, Yang Y, et al. Accuracy of detecting residual disease after neoadjuvant chemoradiotherapy for esophageal squamous cell carcinoma (preSINO trial): a prospective multicenter diagnostic cohort study. BMC Cancer 2020;20:194.

16. Gkotzamanidou M, Sfikakis PP, Kyrtopoulos SA, et al. Chromatin structure, transcriptional activity and DNA repair efficiency affect the outcome of chemotherapy in multiple myeloma. Br J Cancer 2014;111:1293-304.

17. Thompson AK, Kelley BF, Prokop LJ, et al. Risk factors for cutaneous squamous cell carcinoma recurrence, metastasis, and disease-specific death: a systematic review and meta-analysis. JAMA Dermatol 2016;152:419-28.

18. Barbetta A, Sihag S, Nobel T, et al. Patterns and risk of recurrence in patients with esophageal cancer with a pathologic complete response after chemoradiotherapy followed by surgery. J Thorac Cardiovasc Surg 2019;157:1249-59.e5.

19. Asano H, Kojima K, Ogino N, et al. Postoperative recurrence and risk factors of colorectal cancer perforation. Int J Colorectal Dis 2017;32:419-24.

20. He H, Tian D, Guo J, et al. DNA damage response in peritumoral regions of oesophageal cancer microenvironment. Carcinogenesis 2013;34:139-45.

21. Yousif NG, Al-Amran FG, Hadi N, et al. Expression of IL-32 modulates NF- $\kappa$ B and p38 MAP kinase pathways in human esophageal cancer. Cytokine 2013;61:223-7.

22. Crumley AB, Going JJ, Hilmy M, et al. Interrelationships between tumor proliferative activity, leucocyte and macrophage infiltration, systemic inflammatory response, and survival in patients selected for potentially curative resection for gastroesophageal cancer. Ann Surg Oncol 2011;18:2604-12. 
23. Haisley KR, Laird AE, Nabavizadeh N, et al. Association of intervals between neoadjuvant chemoradiation and surgical resection with pathologic complete response and survival in patients with esophageal cancer. JAMA Surg 2016;151:e162743.

24. Fassan M, Cavallin F, Guzzardo V, et al. PD-L1 expression, CD8+ and CD4+ lymphocyte rate are predictive of pathological complete response after neoadjuvant chemoradiotherapy for squamous cell cancer of the thoracic esophagus. Cancer Med 2019;8:6036-48.

25. Tabachnyk M, Distel LV, Büttner M, et al.

Radiochemotherapy induces a favourable tumour infiltrating inflammatory cell profile in head and neck cancer. Oral Oncol 2012;48:594-601.

26. Schurink B, Seesing MFJ, Goense L, et al. ypT0N+ status in oesophageal cancer patients: Location of residual metastatic lymph nodes with regard to the neoadjuvant radiation field. Eur J Surg Oncol 2019;45:454-9.

27. Blackham AU, Yue B, Almhanna K, et al. The prognostic value of residual nodal disease following neoadjuvant chemoradiation for esophageal cancer in patients with complete primary tumor response. J Surg Oncol 2015;112:597-602.

28. Faiz Z, Kats-Ugurlu G, Mul VEM, et al. Locoregional residual esophageal cancer after neo-adjuvant chemoradiotherapy and surgery regarding anatomic site and radiation target fields: a histopathologic evaluation study. Ann Surg 2020. [Epub ahead of print]. doi: 10.1097/ SLA.0000000000004242.

29. van Rossum PSN, van Hillegersberg R, Meijer GJ, et al. Residual esophageal cancer after neoadjuvant chemoradiotherapy frequently involves the mucosa and submucosa. Ann Surg 2015;262:e83-4.

30. Schneider PM, Baldus SE, Metzger R, et al. Histomorphologic tumor regression and lymph node metastases determine prognosis following neoadjuvant

Cite this article as: He W, Mao T, Yan J, Leng X, Deng X, Xie Q, Peng L, Liao Q, Scarpa M, Han Y. Moderately differentiated esophageal squamous cell carcinoma has a poor prognosis after neoadjuvant chemoradiotherapy. Ann Transl Med 2021;9(8):706. doi: 10.21037/atm-21-1815 radiochemotherapy for esophageal cancer: implications for response classification. Ann Surg 2005;242:684-92.

31. Hermann RM, Horstmann O, Haller F, et al. Histomorphological tumor regression grading of esophageal carcinoma after neoadjuvant radiochemotherapy: which score to use? Dis Esophagus 2006;19:329-34.

32. Chirieac LR, Swisher SG, Ajani JA, et al. Posttherapy pathologic stage predicts survival in patients with esophageal carcinoma receiving preoperative chemoradiation. Cancer 2005;103:1347-55.

33. Japan Esophageal Society. Japanese classification of esophageal cancer, 11th edition: part I. Esophagus 2017;14:1-36.

34. Mariette C, Piessen G, Briez N, et al. The number of metastatic lymph nodes and the ratio between metastatic and examined lymph nodes are independent prognostic factors in esophageal cancer regardless of neoadjuvant chemoradiation or lymphadenectomy extent. Ann Surg 2008;247:365-71.

35. Noble F, Lloyd MA, Turkington R, et al. Multicentre cohort study to define and validate pathological assessment of response to neoadjuvant therapy in oesophagogastric adenocarcinoma. Br J Surg 2017;104:1816-28.

36. Semenkovich TR, Subramanian M, Yan Y, et al. Adjuvant therapy for node-positive esophageal cancer after induction and surgery: a multisite study. Ann Thorac Surg 2019;108:828-36.

37. Gamboa AM, Kim S, Force SD, et al. Treatment allocation in patients with early-stage esophageal adenocarcinoma: prevalence and predictors of lymph node involvement. Cancer 2016;122:2150-7.

38. Nobel TB, Livschitz J, Xing XX, et al. Surveillance implications of recurrence patterns in early nodenegative esophageal adenocarcinoma. Ann Thorac Surg 2019;108:1640-7. 